\title{
Method based on atomic photoionization for spot-size measurement on focused soft $x$-ray free-electron laser beams
}

\author{
A. A. Sorokin, ${ }^{\text {a) }}$ A. Gottwald, A. Hoehl, U. Kroth, H. Schöppe, G. Ulm, and M. Richter ${ }^{\text {b) }}$ \\ Physikalisch-Technische Bundesanstalt, Abbestrasse 2-12, 10587 Berlin, Germany \\ S. V. Bobashev, I. V. Domracheva, and D. N. Smirnov \\ Ioffe Physico-Technical Institute, Polytekhnicheskaya 26, 194021 St. Petersburg, Russia \\ K. Tiedtke, S. Düsterer, J. Feldhaus, U. Hahn, U. Jastrow, M. Kuhlmann, T. Nunez, \\ E. Plönjes, and R. Treusch \\ Deutsches Elektronen-Synchrotron, Notkestrasse 85, 22603 Hamburg, Germany
}

(Received 10 May 2006; accepted 13 October 2006; published online 29 November 2006)

\begin{abstract}
A method has been developed and applied to measure the beam waist and spot size of a focused soft $\mathrm{x}$-ray beam at the free-electron laser FLASH of the Deutsches Elektronen-Synchrotron in Hamburg. The method is based on a saturation effect upon atomic photoionization and represents an indestructible tool for the characterization of powerful beams of ionizing electromagnetic radiation. At the microfocus beamline BL2 at FLASH, a full width at half maximum focus diameter of (15 \pm 2$) \mu \mathrm{m}$ was determined. (C) 2006 American Institute of Physics. [DOI: 10.1063/1.2397561]
\end{abstract}

Recent progress in developing pulsed high-power vacuum ultraviolet (VUV) and soft x-ray uv (XUV) sources, such as higher-harmonic generation (HHG) sources $^{1}$ and free-electron lasers (FELs), ${ }^{2}$ has opened the door to extended research on nonlinear interaction of electromagnetic radiation with matter from the optical region to shorter wavelengths. When focused into a spot of a few micrometers in diameter, the radiation can reach peak irradiance levels of more than $10^{13} \mathrm{~W} \mathrm{~cm}^{-2}$ where nonlinear effects such as atomic multiphoton ionization occur. ${ }^{3-6} \mathrm{~A}$ key point for the understanding and theoretical description of nonlinear processes is, in general, their dependence on irradiance. Therefore, among other quantities such as pulse energy and duration, spot-size determination of focused high-intensity VUV and XUV radiation is mandatory.

Recently, two conventional methods have been applied to measure the spot size of focused HHG beams, namely, the knife-edge $^{7}$ and the fluorescence screen technique. ${ }^{1,8-10}$ Both methods provide information on two-dimensional photon intensity distribution with a spatial resolution of 1 to $2 \mu \mathrm{m}$. The possibility of applying these techniques to FELs is, however, limited. The FEL pulse energy levels of VUV and XUV radiation may be some orders of magnitude higher than those of HHG sources and can cause radiation damage on fluorescence screens and, in general, on any solid irradiated surface. Moreover, due to its statistical nature, a beam of FEL radiation based on self-amplified spontaneous emission ${ }^{2}$ may strongly fluctuate from shot to shot, perpendicular to the propagation axis, and requires spot-size measurements which do not depend on the beam position. In this context, we describe a method which has been used to determine the beam waist and spot size of a focused beam at the XUV-FEL facility FLASH of the Deutsches Elektronen-Synchrotron in Hamburg. ${ }^{11}$ It is based on a saturation effect upon photoionization of a rare gas and manifests itself by a sublinear increase in the ion yield as a function of the photon number per

\footnotetext{
${ }^{a)}$ Also at Ioffe Physico-Technical Institute, Polytekhnicheskaya 26, 194021 St. Petersburg, Russia.

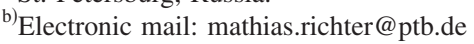

pulse. It is due to a considerable reduction in the number of target atoms within the interaction zone by ionization with a single photon pulse and becomes stronger with decreasing beam cross section. The method is indestructible and not affected by fluctuations of the beam position. Moreover, it can easily be realized in any ionization chamber by introducing a (rare) gas and detecting the photoionization signal as a function of the absolute photon number per pulse.

Our measurements were performed at the microfocus beamline BL2 at FLASH with several series of subsequent photon pulses of $25 \mathrm{fs}$ duration, separated by $1 \mu \mathrm{s}$, with up to $10^{12}$ photons, at a photon energy of $38 \mathrm{eV}$. An ellipsoidal mirror was used for focusing. The photoionization (PI) setup consists of a vacuum chamber with a conventional time-offlight (TOF) spectrometer (Fig. 1) homogeneously filled with the target gas. Along the photon beam, the spectrometer has an acceptance length of $1.0 \mathrm{~mm}$ and, for measurements at different beam sizes, can be moved $\pm 2 \mathrm{~cm}$ around the focus position. At a pressure around $10^{-3} \mathrm{~Pa}$, applied and measured with the help of a calibrated spinning rotor gauge, the target gas is almost transparent, allowing the absolute photon number per pulse to be determined online $50 \mathrm{~cm}$ behind the focus. For this purpose, we used a gas-monitor detector (GMD) which has recently been developed ${ }^{12}$ and established as a reliable intensity monitor for VUV-FEL radiation. ${ }^{2}$ The GMD, again, is based on the photoionization of a rare gas. Its calibration was performed at the electron storage ring

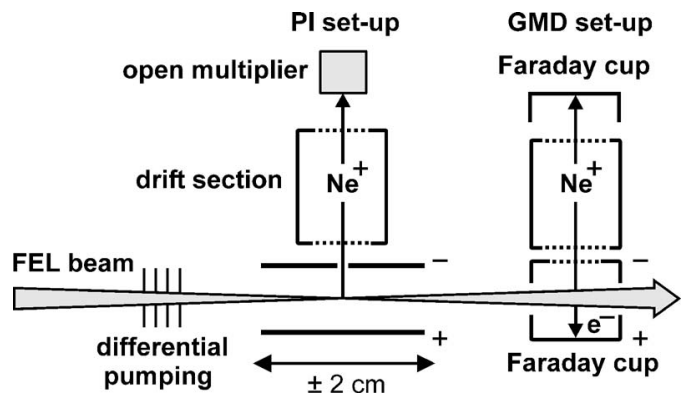

FIG. 1. Schematic diagram of the experimental setup. 


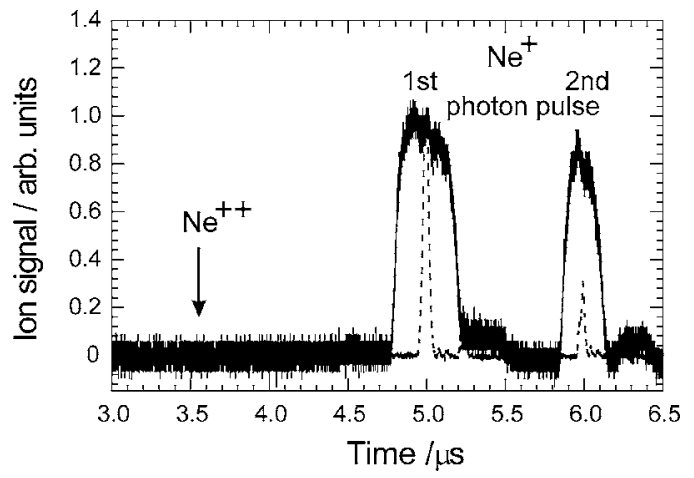

FIG. 2. Two single-shot TOF spectra of neon (Ne), obtained from two subsequent FEL pulses, at low (dashed line) and high (solid line) numbers of ions generated per pulse.

BESSY II in the Radiometry Laboratory of the PhysikalischTechnische Bundesanstalt, Germany's National Metrology Institute, with a relative standard uncertainty of $10 \% .^{12}$ The GMD almost resembles the PI setup. In both cases, photogenerated electrons and ions are extracted by a homogeneous electric field perpendicular to the photon beam. Electron and ion detection in the GMD is realized with the help of chargecollecting Faraday cups. For the ion TOF spectrometer of the PI setup, an open electron multiplier with an active area of $2 \times 2 \mathrm{~cm}^{2}$ was used, optimized and tested for absolute ion collection/detection efficiency and linearity for up to $10^{6}$ ions detected per pulse. During measurement, GMD and ion TOF single-shot signals were recorded and pulse resolved simultaneously by means of a digital oscilloscope using the FEL bunch clock for triggering. Neon was chosen as the target gas because at a photon energy of $38 \mathrm{eV}$, the photoionization of neon is restricted to the generation of singly charged ions only but with a considerably high cross section of $\sigma$ $=(8.6 \pm 0.3) \times 10^{-18} \mathrm{~cm}^{2} .^{13}$

Figure 2 shows two TOF spectra of neon taken from two subsequent FEL pulses. The absence of any signal from doubly charged ions indicates that our measurements were not affected by higher-order effects such as photoionization by higher harmonics of the FEL or atomic two-photon processes. Figure 3 demonstrates the mentioned saturation effect for the single photoionization of neon. Shown is the sublinear dependence of the number of $\mathrm{Ne}^{+}$ions created per pulse on the absolute photon number per pulse.

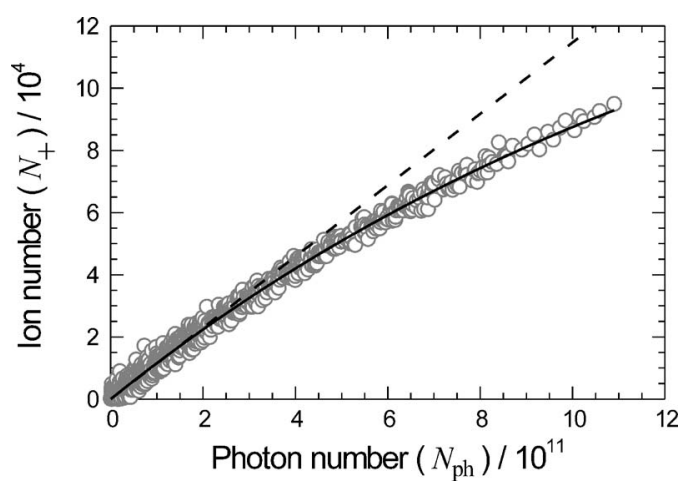

FIG. 3. Absolute number of $\mathrm{Ne}^{+}$ions created per FEL pulse as a function of the absolute photon number per pulse. The solid line represents a fit curve according to Eq. (2). The dashed line depicts a linear dependency for

collection/detection. However, below $2 \times 10^{-3} \mathrm{~Pa}$, ion detec-
Downloaded 19 Mar 2007 to 131.169.95.147. Redistribution subject to AIP license or copyright, see http://apl.aip.org/apl/copyright.jsp

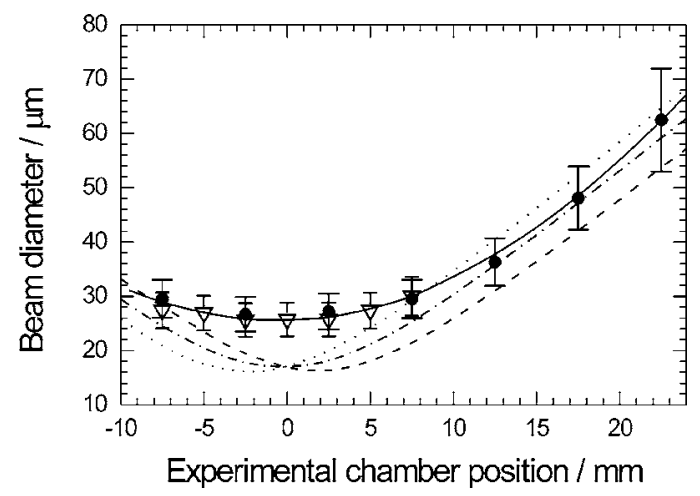

FIG. 4. $2 \sigma$ value of the photon beam diameter $d_{2 \sigma}=(4 A / \pi)^{1 / 2}$, measured at different positions of the experimental chamber along the photon beam around the focus and at a target gas pressure of $8 \times 10^{-4} \mathrm{~Pa}(\bullet)$ and 2 $\times 10^{-3} \mathrm{~Pa}(\nabla)$. Data evaluation is based on saturation in the single photoionization of neon according to Fig. 3 and Eq. (2). The solid line represents a polynomial fit curve. The nonsolid lines display ray-trace simulations for the horizontal (dashed line) and vertical directions (dotted line) and the mean of both (dashed-dotted line).

A corresponding expression for the number of ions $N_{+}$ generated per photon pulse may be derived from the general definition of a photoionization cross section $\sigma$,

$$
N_{+}\left(H_{\mathrm{ph}}\right)=\iint n z\left(1-\exp \left(-\sigma H_{\mathrm{ph}}(x, y)\right)\right) d x d y .
$$

Here, $n$ represents the target gas density and $z$ the length of the interaction zone along the photon beam accepted by the TOF spectrometer. $H_{\mathrm{ph}}$ denotes the photon exposure, i.e., the photon number per pulse and unit area: $H_{\mathrm{ph}}=d N_{\mathrm{ph}} / d A$. For a homogeneous photon distribution within a beam cross section $A$, Eq. (1) is transformed to an ordinary exponential saturation function,

$$
\begin{aligned}
N_{+}\left(N_{\mathrm{ph}}\right) & =N\left(1-\exp \left(-\sigma \frac{N_{\mathrm{ph}}}{A}\right)\right) \\
& \approx n z \sigma N_{\mathrm{ph}}\left(1-\frac{1}{2}\left(\sigma \frac{N_{\mathrm{ph}}}{A}\right)+\frac{1}{6}\left(\sigma \frac{N_{\mathrm{ph}}}{A}\right)^{2}\right) .
\end{aligned}
$$

Here, $N=n z A$ is the initial number of atoms within the interaction zone. The third-order approximation in Eq. (2) can be shown to be almost valid also in the case of a Gaussian beam profile, with $A$ equal to the $2 \sigma$ area. Only the coefficient $1 / 6$ has to be substituted by $2 / 9$ in this case.

Equation (2) demonstrates that the saturation effect due to vanishing targets is determined by the argument $\sigma N_{\mathrm{ph}} / A$ which should - for the sake of reliability of our method - be at least in the order of 0.1 to 1 . Thus, fitting experimental data for $N_{+}\left(N_{\mathrm{ph}}\right)$ given on an absolute $N_{\mathrm{ph}}$ scale (Fig. 3) by the saturation curve of Eq. (2) yields, together with the known single photoionization cross section $\sigma$ of neon, the beam cross section $A$ and, by that, the focus diameter as a fit parameter. The result does not represent a single-shot determination of $A$ but an average value for about 1000 subsequent FEL pulses. Figure 4 summarizes the evaluation of a set of those measurements in order to find the focus position, i.e., the local minimum of the beam diameter, while moving the experimental chamber along the photon beam. The measurements were repeated at different target gas pressuresand thus signal intensities - in order to investigate the influence of other saturation effects, e.g., due to incomplete ion collection/detection. However, below $2 \times 10^{-3} \mathrm{~Pa}$, ion detec- 
tion has been confirmed to be linear and high numbers of ions generated per pulse lead to line broadening due to space charge effects and Coulomb repulsion in the respective ion spectra only (Fig. 2), whereas the beam waist measurements were not affected by the target gas pressure (Fig. 4).

As a further result, assuming a Gaussian intensity distribution, the full width at half maximum (FWHM) focus diameter $d_{\mathrm{FWHM}}=d_{2 \sigma}(\ln 2 / 2)^{1 / 2}=(2 \ln 2 \times A / \pi)^{1 / 2}$ at BL2 has been found to be $15 \pm 2 \mu \mathrm{m}$, with a beam divergence of about $3 \mathrm{mrad}$. The absolute measurement uncertainty for the focus diameter results from the uncertainty of the GMD calibration, the standard deviation of the fit curves from the experimental saturation data (Fig. 3), and, in combination with the beam divergence, the spatial resolution of the measurements along the photon beam due to the acceptance length of the TOF spectrometer. The latter may be reduced for smaller focus sizes by reducing the width of the spectrometer entrance slit. Figure 4 shows also the results of ray-trace simulations using the SHADOW code. ${ }^{14}$ While the agreement is almost perfect for distances greater than $10 \mathrm{~mm}$ from the focus position, the beam diameter within the focus region seems to be slightly larger than predicted, which might be explained by minor misalignment and only partially known source parameters.

In conclusion, we have demonstrated that beam waist and spot-size determination on pulsed high-power VUV- and XUV-FEL beams may be realized in an indestructible manner by gas-phase experiments. At the microfocus beamline BL2 of the XUV-FEL facility FLASH in Hamburg, a FWHM focus diameter of $15 \mu \mathrm{m}$ has been measured. With $10^{12}$ XUV photons within one single FEL pulse, our method is restricted to beam diameters below $100 \mu \mathrm{m}$ since it is based on a saturation effect upon atomic photoionization. For harder x-rays at $10 \mathrm{keV}$ photon energy, for example, the beam diameter should be even smaller than $10 \mu \mathrm{m}$ due to the generally much lower photoionization cross section values. Since the method becomes more sensitive for smaller beam diameters, it represents, however, a powerful tool for submicrometer spot-size determination also at future hard x-ray laser sources.
${ }^{1}$ H. Mashiko, A. Suda, and K. Midorikawa, Appl. Opt. 45, 573 (2006).

${ }^{2}$ J. Feldhaus, J. Arthur, and J. B. Hastings, J. Phys. B 38, S799 (2005).

${ }^{3}$ H. Wabnitz, L. Bittner, A. R. B. de Castro, R. Döhrmann, P. Gürtler, T. Laarmann, W. Laasch, J. Schulz, A. Swiderski, K. von Haeften, T. Möller, B. Faatz, A. Fateev, J. Feldhaus, C. Gerth, U. Hahn, E. Saldin, E. Schneidmiller, K. Sytchev, K. Tiedtke, R. Treusch, and M. Yurkov, Nature (London) 420, 482 (2002).

${ }^{4}$ H. Wabnitz, A. R. B. de Castro, P. Gürtler, T. Laarmann, W. Laasch, J. Schulz, and T. Möller, Phys. Rev. Lett. 94, 023001 (2005).

${ }^{5}$ N. Miyamoto, M. Kamei, D. Yoshitomi, T. Kanai, T. Sekikawa, T. Nakajima, and S. Watanabe, Phys. Rev. Lett. 93, 083903 (2004).

${ }^{6}$ Y. Nabekawa, H. Hasegawa, E. J. Takahashi, and K. Midorikawa, Phys. Rev. Lett. 94, 043001 (2005).

${ }^{7}$ L. Le Déroff, P. Salières, and B. Carré, Opt. Lett. 23, 1544 (1998).

${ }^{8}$ D. Yoshitomi, T. Shimizu, T. Sekikawa, and S. Watanabe, Opt. Lett. 27, 2170 (2002).

${ }^{9}$ C. Valentin, D. Douillet, S. Kazamias, Th. Lefrou, G. Grillon, F. Augé, G. Mullot, Ph. Balcou, P. Mercère, and Ph. Zeitoun, Opt. Lett. 28, 1049 (2003).

${ }^{10}$ H. Mashiko, A. Suda, and K. Midorikawa, Opt. Lett. 29, 1927 (2004).

${ }^{11}$ V. Ayvazyan, N. Baboi, J. Bähr, V. Balandin, B. Beutner, A. Brandt, I. Bohnet, A. Bolzmann, R. Brinkmann, O. I. Brovko, J. P. Carneiro, S. Casalbuoni, M. Castellano, P. Castro, L. Catani, E. Chiadroni, S. Choroba, A. Cianchi, H. Delsim-Hashemi, G. Di Pirro, M. Dohlus, S. Düsterer, H. T. Edwards, B. Faatz, A. A. Fateev, J. Feldhaus, K. Flöttmann, J. Frisch, L. Fröhlich, T. Garvey, U. Gensch, N. Golubeva, H.-J. Grabosch, B. Grigoryan, O. Grimm, U. Hahn, J. H. Han, M. v. Hartrott, K. Honkavaara, M. Hüning, R. Ischebeck, E. Jaeschke, M. Jablonka, R. Kammering, V. Katalev, B. Keitel, S. Khodyachykh, Y. Kim, V. Kocharyan, M. Körfer, M. Kollewe, D. Kostin, D. Krämer, M. Krassilnikov, G. Kube, L. Lilje, T. Limberg, D. Lipka, F. Löhl, M. Luong, C. Magne, J. Menzel, P. Michelato, V. Miltchev, M. Minty, W. D. Möller, L. Monaco, W. Müller, M. Nagl, O. Napoly, P. Nicolosi, D. Nölle, T. Nuñez, A. Oppelt, C. Pagani, R. Paparella, B. Petersen, B. Petrosyan, J. Pflüger, P. Piot, E. Plönjes, L. Poletto, D. Proch, D. Pugachov, K. Rehlich, D. Richter, S. Riemann, M. Ross, J. Rossbach, M. Sachwitz, E. L. Saldin, W. Sandner, H. Schlarb, B. Schmidt, M. Schmitz, P. Schmüser, J. R. Schneider, E. A. Schneidmiller, H.-J. Schreiber, S. Schreiber, A. V. Shabunov, D. Sertore, S. Setzer, S. Simrock, E. Sombrowski, L. Staykov, B. Steffen, F. Stephan, F. Stulle, K. P. Sytchev, H. Thom, K. Tiedtke, M. Tischer, R. Treusch, D. Trines, I. Tsakov, A. Vardanyan, R. Wanzenberg, T. Weiland, H. Weise, M. Wendt, I. Will, A. Winter, K. Wittenburg, M. V. Yurkov, I. Zagorodnov, P. Zambolin, and K. Zapfe, Eur. Phys. J. D 37, 297 (2006).

${ }^{12}$ M. Richter, A. Gottwald, U. Kroth, A. A. Sorokin, S. V. Bobashev, L. A. Shmaenok, J. Feldhaus, Ch. Gerth, B. Steeg, K. Tiedtke, and R. Treusch, Appl. Phys. Lett. 83, 2970 (2003).

${ }^{13}$ J. M. Bizau and F. J. Wuilleumier, J. Electron Spectrosc. Relat. Phenom. 71, 205 (1995).

${ }^{14}$ B. Lai and F. Cerrina, Nucl. Instrum. Methods Phys. Res. A 246, 337 (1986). 